\title{
Interaction between urokinase receptor and heat shock protein MRJ enhances cell adhesion
}

\author{
CHARLES EDO DE BOCK ${ }^{1,2^{*}}$, ZHEN LIN $^{1 *}$, AHMED H. MEKKAWY ${ }^{1}$, JENNIFER A. BYRNE ${ }^{3}$ and YAO WANG ${ }^{1,4}$ \\ ${ }^{1}$ Division of Critical Care and Surgery, St. George Hospital, University of New South Wales, Kogarah, Sydney, NSW 2217; \\ ${ }^{2}$ Leukaemia Foundation Research Laboratory, The Queensland Institute of Medical Research, Brisbane, QLD 4029; \\ ${ }^{3}$ Children's Cancer Research Unit, The Children's Hospital at Westmead, NSW 2145, Australia; ${ }^{4}$ The State Key \\ Laboratory of Pharmaceutical Biotechnology, Nanjing University, Nanjing 210093, P.R. China
}

Received December 15, 2009; Accepted January 18, 2010

DOI: 10.3892/ijo_00000598

\begin{abstract}
The urokinase-type plasminogen activator receptor (UPAR) has diverse biological functions including roles in proteolysis, cell adhesion and cellular signaling. We identified a heat shock protein MRJ (DNAJB6) as a novel uPAR-interacting protein in a yeast two-hybrid screen and confirmed the interaction and co-localization by GST-pull down assays, and co-immunoprecipitation in cells transfected with MRJ. Endogenous interaction between UPAR and MRJ was also detected in breast cancer MDA-MB-231 cells. Deletion mapping demonstrated that the $\mathrm{C}$-terminal region of MRJ is required to mediate its interaction with UPAR. To understand the biological function of the uPAR-MRJ complex, we determined whether MRJ regulated UPAR mediated adhesion to vitronectin in human embryonic kidney (HEK) 293 cells stably transfected with uPAR. After transfection with full length MRJ, there was a $50 \%$ increase in cell adhesion compared to the mock transfected control $(\mathrm{p}<0.01)$. This increase in adhesion is dependent on the uPAR/full length MRJ interaction as cells transfected with the mutant construct
\end{abstract}

Correspondence to: Dr Charles E. De Bock, Leukaemia Foundation Research Laboratory, Queensland Institute of Medical Research, Herston, QLD, Australia

E-mail: charlesB@qimr.edu.au

cedebock@hotmail.com

Dr Yao Wang, St. George Clinical School, University of New South Wales, NSW 2217, Australia

E-mail: yao_wang_au@yahoo.com

*Contributed equally

Abbreviations: ECM, extracellular matrix; GPI, glycosyl phosphatidylinositol; HSP, heat shock protein; MRJ, mammalian relative DnaJ; PBS, phosphate-buffered saline; SDS, sodium dodecyl sulfate; uPA, urokinase-type plasminogen activator; uPAR, uPA receptor

Key words: heat shock protein MRJ, protein interaction, vitronectin containing only $\mathrm{N}$-terminal region or $\mathrm{C}$-terminal region of MRJ had no increase in cell adhesion. The observed increase in adhesion to vitronectin by MRJ was also blocked by an anti-uPAR domain I antibody suggesting that the induced adhesion is at least in part contributed by uPAR on the cell surface. These data provide a novel mechanism by which uPAR plays a role in cell adhesion to vitronectin.

\section{Introduction}

Urokinase-type plasminogen activator (uPA) receptor (uPAR) has been implicated in multiple cellular pathways, including proteolysis, cell adhesion and signal transduction. The uPAR protein sequence has three repeats which form three homologous domains with $\sim 90$ amino acids each. These have been designated DI, DII and DIII from the N-terminal end (1). These domains are homologous to the CD59/Ly6 family (2) and have two short linker regions between DI and DII, as well as DII and DIII. Cleavage of UPAR by both chymotrypsin and uPA occurs in the linker region between DI and DII at residues 87 and 84 , respectively, to result in a cleaved form of UPAR (3). There is also a soluble variant of UPAR that has been found in the media of cell lines and body fluids from cancer patients. This soluble form may be either the full-length receptor or a truncated form DIIDIII (4). Binding of uPA to its receptor can activate signal transduction pathways including the Ras/ERK pathway by a mechanism that requires FAK, Src, and Shc activation and induction of transient c-fos gene expression $(5,6)$. In addition to the binding of uPA, several proteins have been found to be associated with UPAR functions or signalling pathways. These include integrins $\beta 1 / 2 / 3$, vitronectin, cytokeratins $8 / 18$, EGFR (epidermal growth factor receptor), PDGFR (platelet derived growth factor receptor), LDLR- (low density lipoprotein receptor-) related protein, FPR (FMLP-receptor), UPARAP and G-protein coupled receptors FPRL1 (FMLP-receptor-like protein 1). These interactions with uPAR result in various functional consequences depending on the specific interacting protein (4,7-9). For example, integrin $B 1$ interacts with uPAR and forms a uPA-uPAR- $\alpha 5 \beta 1$ complex initiating intracellular signalling (10). Vitronectin binds to UPAR, and once phosphorylated, regulates uPA-dependent cell adhesion. However, 
the numerous and varied roles of uPAR in cell adhesion, migration, proliferation, angiogenesis and cancer metastasis are not completely explained by identified known protein interactions. Hence we hypothesized there are still additional and as yet unidentified proteins that interact with uPAR. These additional interacting proteins could bind to any of the multiple forms of UPAR as described, which can be located either as a GPI-anchored protein on the cell surface, as a cleaved soluble form, or within intracellular vesicles in the cytoplasm after internalization. In addition, recent crystalline structure modelling of uPAR has found the external surface of UPAR to be free to interact with other proteins even when bound to uPA (11). The yeast two-hybrid system has been used widely in studying intracellular protein-protein interactions. However, more recently, it has also been used successfully to investigate interactions between extracellular proteins such as perlecan (12), thrombospondin (13), EMILIN (14) and matrix metalloproteinase 2 (15). Furthermore, a member of the plasminogen activation system PAI-1 has been used directly as a bait on two occasions and found to interact with $\alpha 1$-acid glycoprotein (16) and $\alpha$-actinin-4 (17). To identify novel UPAR partners and find functional significance of the interaction, we screened a breast cancer cDNA library with several bait constructs using a yeast two-hybrid approach. Here we show that the heat shock protein MRJ (mammalian relative DnaJ) interacts functionally with UPAR to increase uPAR mediated cell adhesion to vitronectin.

\section{Materials and methods}

Materials, vectors and yeast strains. The yeast vectors pGBD-B and pACT2-B and yeast strains PJ69-4A and PJ69-4 $\alpha$ were kindly provided by Dr David Markie (Department of Pathology, Dunedin School of Medicine, New Zealand). The pRK5-myc-MRJ-F, pRK5-myc-MRJ-N1, pRK5-myc-MRJN2, pRK5-myc-MRJ-C, pACT2-MRJ-C and pACT $\alpha-M R J-F$ were all kindly provided by Masaki Inagaki and Ichiro Izawa (Aichi Cancer Center Research Institute, Japan). The stable uPAR transfected human embryonic kidney (HEK) 293 cells were kindly provided by Dr Ying Wei (University of California, San Francisco). The anti-uPAR antibodies (nos. 3931, AF807, MAB807) were purchased from American Diagnostica Inc or R\&D Systems, Inc. The mouse monoclonal anti-uPAR antibodies R2 and R3 were kindly provided by Dr Niels Behrendt (Copenhagen, Denmark). The mouse antihuman MRJ and anti-human DNAJB4 antibodies were purchased from Abnova Corp. (Taiwan). The anti-myc antibody and anti- $\beta$ actin antibody were purchased from SigmaAldrich.

Expression cDNA library construction and cloning of open reading frames of $u P A R$ baits. A human breast carcinoma cDNA library (18) was transformed on a large scale into PJ69-4 $\alpha$ to construct a pre-transformed library of $4.7 \times 10^{7}$ independent yeast colonies. Five UPAR baits were designed based on the UPAR full-length cDNA sequence and amplified by PCR using gene-specific primers that added short sequence tags (Fig. 1A) (19). The resulting DNA fragments were cotransformed into yeast host PJ69-4A with BamHI linearized pGBD-B vector DNA and plated with selection for the TRPI gene. Individual colonies were tested by PCR for the presence of an insert with correct size.

Yeast two-hybrid screening and cloning of MRJ preys. A mating strategy described previously was used for the yeast two-hybrid screening (19). Briefly, library transformed PJ69-4 $\alpha$ was grown in YPAD media. Bait plasmid transformed PJ69-4A yeast was grown overnight in a rich Trp-media (SD+C supplemented with $100 \mu \mathrm{g} / \mathrm{ml}$ leucine, $20 \mu \mathrm{g} / \mathrm{ml}$ histidine, $20 \mu \mathrm{g} / \mathrm{ml}$ uracil and $32 \mu \mathrm{g} / \mathrm{ml}$ adenine). A total of $5 \times 10^{8}$ yeast cells of bait and library were used per screen. The resulting diploids were tested for HIS3, ADE2 and LacZ reporter gene activation. Secondary confirmation of interactions were done as described previously (19). Full-length pRK5-myc-MRJ-F, pRK5-mycMRJ-N1, pRK5-myc-MRJ-N2, pRK5-myc-MRJ-C, pACT2MRJ-C and pACT $\alpha-M R J-F$ have been described previously (20).

Yeast two-hybrid cross mating. The pACT2-MRJ-C and pACT $\alpha-M R J-F$ plasmids were transformed into PJ69-4 $\alpha$ and then mated against the five UPAR bait constructs transformed in PJ69-4A yeast as described (21).

In vitro transcription and translation and GST-pull down assay. The TNT ${ }^{\circledR}$ T7 Quick coupled Rabbit Reticulocyte Transcription/Translation System (Promega, Madison, WI, USA) was used to label MRJ with $\left[{ }^{35} \mathrm{~S}\right]$-methionine. PCR reaction products for DI, DII, DIII, DIIDIII and DIDIIDIII UPAR were used to clone into the N-terminal glutathione S-transferase (GST) containing pDEST $^{\mathrm{TM}} 15$ plasmid using the Gateway ${ }^{\circledR}$ System (Invitrogen, Carlsbad, CA, USA). GST-tagged protein was expressed and purified in BL21-AI ${ }^{\mathrm{TM}}$ $E$. coli using standard protocol and purified $>50 \%$ glutathioneagarose bead slurry. Equivalent amount of GST-tagged UPAR protein was incubated with labelled MRJ protein and washed with pull down buffer [150 mM NaCl, $20 \mathrm{mM}$ Tris- $\mathrm{HCl}$ (pH 7.5), 0.1\% NP-40, $2.5 \mathrm{mg} / \mathrm{ml} \mathrm{BSA,} 0.01 \%$ 2-ME, $1.2 \mathrm{mM} \mathrm{PMSF}$. Bound protein was eluted with $3 \mathrm{X}$ SDS loading buffer and separated by SDS-PAGE. The labelled bound protein was visualised by phosphor imager (Bio-Rad) and analyzed using the Quantity One Software (Bio-Rad).

Northern blotting. RNA was isolated from cells with TRIzol Reagent using standard protocols of Invitrogen. Northern blot analysis was carried out as described previously (22).

Cell culture, plasmid transfection and $\beta$-galactosidase assay. The human breast cancer MDA-MB-231 and HEK293 cell lines were maintained in DMEM medium supplemented with $10 \%$ fetal calf serum (FCS), and $1 \%$ antibiotics. The stable transfected HEK293/uPAR cell line was cultured in the presence of $0.9 \mathrm{mg} / \mathrm{ml} \mathrm{G} 418$ (23) (Geneticin). Transfections were performed using Lipofectamine ${ }^{\mathrm{TM}} 2000$ as per manufacturer's instructions (Invitrogen). Briefly, cells were transfected with recombinant or control vector and also pRSV$ß$-galactosidase plasmid to control for transfection efficiency. Whole cell lysates were prepared using reporter lysis buffer and $\beta$-galactosidase activity was measured using standard protocols (Promega). 
A

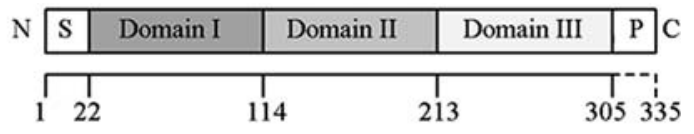

1. UPAR-DIDIIDIII $\square$

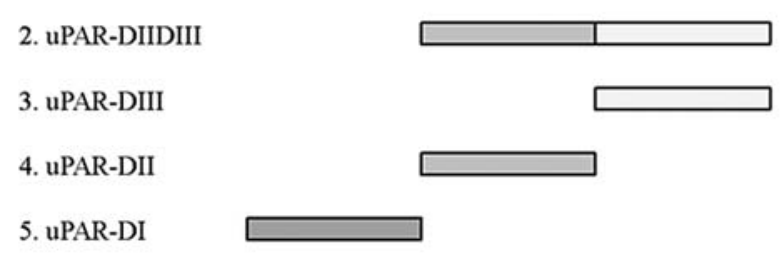

B

MRJ Protein \begin{tabular}{|l|l|lr}
\hline Jdomain & G/F & & \\
\hline & 74 & 119 & \\
\hline
\end{tabular}

\begin{tabular}{lcc} 
& \multicolumn{8}{c}{} & \\
\hline MRJ Prey sequence & Positive MRJ Clones & Total Positive Clones \\
\hline Bait & 6 & 17 \\
uPAR-DIDIIDIII & 1 & 2 \\
uPAR-DI & 17 \\
\hline
\end{tabular}

Figure 1. Identification of MRJ as a binding protein of uPAR using the yeast two-hybrid system. (A) Schematic diagram illustrating the five uPAR domain combinations used as baits, compared with the full-length uPAR amino acid sequence, where numbers refer to the amino acid positions. The $\mathrm{N}$-terminal signal peptide (S) and C-terminal pro-peptide $(\mathrm{P})$ were omitted. The glycine residue at amino acid position 305 involved in GPI-anchor attachment was also omitted from the bait construction. (B) The MRJ prey sequence identified in the yeast two-hybrid screen consisted of amino acid residues 81-241. The uPAR-DIDIIDIII and uPAR-DI baits both identified MRJ independently as a putative interacting protein. The number of positive MRJ clones and total positive clones identified with these baits are indicated.

Co-immunoprecipitation (coIP) and Western blotting (WB). HEK293/uPAR cells were transiently transfected with the pRK5-myc-MRJ-F plasmid with Lipofectamine 2000. Following incubation for $48 \mathrm{~h}$, the medium was removed, and the cells washed with PBS, and lysed with IP buffer. Cell lysate was pre-cleared with protein G-agarose (Pierce, Rockford, IL, USA) before undergoing IP with antibodies [rabbit anti-c-myc (C3956, Sigma), goat anti-uPAR (AF807), and their isotype-matched IgG controls]. MDA-MB-231 cells were lysed and treated as above but underwent IP with anti-uPAR, anti-MRJ or anti-DNAJB4 antibodies and their isotype-matched IgG controls. The complex was collected and subjected to WB as described (22). No DTT was added to samples to prevent co-migration of the heavy chain $\mathrm{IgG}$ with the uPAR band (Fig. 5B).

Cell adhesion assay. The assay was performed as described previously with modifications (23). Briefly, 96-well dishes were pre-coated with $2 \mu \mathrm{g} / \mathrm{ml}$ vitronectin or heat-denatured BSA (Sigma) overnight at $4^{\circ} \mathrm{C}$. Plates were rinsed with PBS, and all wells incubated with $2 \%$ heat-denatured BSA to block any uncoated areas. Cells $\left(1.5 \times 10^{5}\right.$ cells/well $)$ were seeded in the coated wells and incubated for $2 \mathrm{~h}$ at $37^{\circ} \mathrm{C}$. After washing the attached cells were fixed with methanol/acetone and stained with $0.1 \%$ crystal violet. The stain was eluted using acetic acid/methanol/water and absorbance measured.
Statistical analysis. All values are expressed as the mean \pm standard deviation and statistical significance calculated using Student's t-tests where appropriate.

\section{Results}

Yeast two-hybrid screening and confirmation of cross-mating between $U P A R$ and MRJ. As shown in Fig. 1A, five different uPAR baits, full-length uPAR (DIDIIDIII), domain II plus III (uPAR-DIIDIII), domain III (uPAR-DIII), domain II (uPARDII) and domain I (uPAR-DI) were constructed and used in subsequent yeast two-hybrid screen. A number of candidate proteins were identified by each bait (data not shown). Among the candidates, the C-terminal end of MRJ was identified by both the uPAR-DI (1/2 positive clones) and uPAR-DIDIIDIII (6/17 positive clones) baits (Fig. 1B). Following this initial identification of MRJ as a putative binding protein of UPAR, a number of deletion mutants based on the full length sequence of MRJ were used (Fig. 2A) to further assess the binding between uPAR and MRJ. To this end, a cross mating approach was undertaken between the yeast containing $\mathrm{C}$-terminal fragment (MRJ-C) and the full-length MRJ (MRJ-F) prey and the five uPAR bait. Fig. 2B shows efficient mating between all five UPAR baits and MRJ-C prey containing strains (streaked in duplicate), as shown by growth on Leu-/Trp- drop out media. When UPAR-DIDIIDIII and MRJ-C were present in the same diploid colonies, there was strong activation of the HIS3, ADE2 and $L a c Z$ reporter genes. This activity was reduced for the UPAR-DI bait. There was only residual reporter gene activation in the UPAR-DIDIIDIII/MRJ-F and uPAR-DI/MRJ-F diploids, which may have been due to inefficient mating. There was no detectable reporter gene activation in diploids containing MRJ-F and uPAR-DII, uPAR-DIII or uPAR-DIIDIII. These results suggested that the $\mathrm{UPAR} / \mathrm{MRJ}$ interaction occurred between the C-terminal domain of MRJ and DI or the full length DIDIIDIII uPAR.

UPAR interact with the C-terminal end of MRJ by GST-pull down assay. To confirm the interaction between UPAR and MRJ proteins, an in vitro GST-pull down assay was undertaken. To elucidate whether a particular domain of UPAR was involved in the interaction, different GST-tagged UPAR constructs were incubated with $\left[{ }^{35} \mathrm{~S}\right]$-methionine labelled MRJ-F. Fig. 3A suggested that MRJ-F may bind preferentially with GST-uPAR-DIDIIDIII and GST-uPAR-DI proteins, consistent with our yeast two-hybrid results. A second assay was employed to map the interaction domain in MRJ involved. Four alternate $\left[{ }^{35} \mathrm{~S}\right]$-methionine labelled MRJ constructs were incubated with the GST-uPAR-DIDIIDIII protein or GST alone (Fig. 3B). uPAR-DIDIIDIII bound strongly to MRJ-F and MRJ-C, but weakly to MRJ-N2 and not at all to MRJ-N1, indicating that the MRJ C-terminal domain is required for interactions with uPAR. This result implicates the C-terminal domain of MRJ as the predominant region for the interaction between UPAR and MRJ, with no apparent binding to the $\mathrm{J}$-domain or the adjacent glycine/phenylalanine-rich $(\mathrm{G} / \mathrm{F})$ region.

UPAR and MRJ are co-expressed in cancer cell lines. To test whether UPAR and MRJ are co-expressed and to determine 


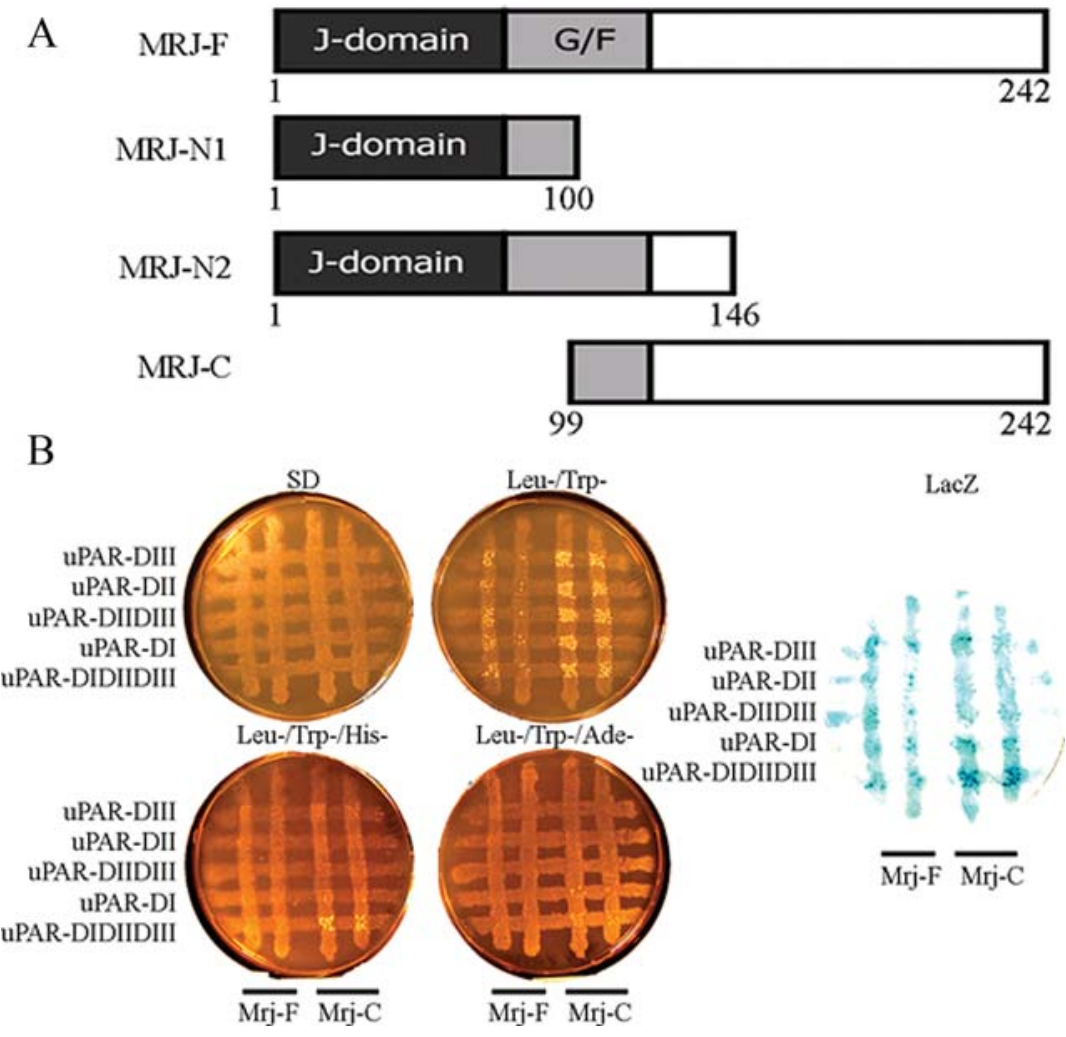

Figure 2. MRJ deletion mutants and confirmation of interaction between uPAR and MRJ using cross-mating. (A) Schematic representation of domain organization of full-length MRJ (MRJ-F; a.a. 1-242) and three mutant MRJ proteins. Numbers refer to amino acid positions. The J domain (a.a. 1-74), the glycine/phenylalanine-rich domain (G/F; a.a. 75-119) and C-terminal region (a.a. 120-242) are indicated. The MRJ deletion mutants MRJ-N1(a.a. 1-100), MRJ-N2 (a.a. 1-146), and MRJ-C (a.a. 99-242) are also indicated. (B) The five uPAR baits were transformed into the yeast strain PJ69-4A, and the MRJ-F and MRJ-C were transformed into PJ69-4 $\alpha$. These were then cross-mated to introduce the two constructs into the same diploid yeast. There was detectable activation of the HIS3, ADE2 and LacZ reporter genes when both MRJ-C and uPAR-DIDIIDIII and uPAR DI were expressed in the same diploid (evidenced by growth on the -Leu/-Trp/-His and -Leu/-Trp/-Ade drop out media and blue colour development with X-gal, respectively). The MRJ-F construct produced low mating efficiency and had undetectable reporter gene activation. The cross-mating was repeated twice and representative data are shown.

a suitable cell line for further studies, we performed mRNA and protein analyses in human cancer cell lines (Fig. 4). High level expression of MRJ and uPAR mRNAs was notable in the MDA-MB-231 cell line compared to other cancer cell lines (Fig. 4A). There are two isoforms of human MRJ, isoform a (2.5 kb transcript variant I, NM_058246) and isoform b (1.6 kb transcript variant II, NM_005494). The variant II differs in the $3^{\prime}$ coding region and has a distinct 3' UTR, compared to transcript variant I. The resulting isoform $\mathrm{b}$ is shorter and distinct, compared to isoform $\mathrm{a}$. It is the shorter transcript variant II that encodes the MRJ isoform $b$ that interacts with uPAR. To determine whether this mRNA expression was reflected at the protein level, Western blot analysis was carried out in HeLa, MDA-MB-231 and 143CTK cell lines. HEK293/uPAR cells transfected with myc-MRJ-F was used as a positive control. The major uPAR protein band occurred at $\sim 55-60 \mathrm{kDa}$, with additional bands at 45 and $35 \mathrm{kDa}$ corresponding to glycosylated, soluble and deglycosylated UPAR forms, respectively. Two endogenous MRJ protein bands were detected with apparent molecular weights of $\sim 32$ and $\sim 45 \mathrm{kDa}$ (Fig. 4B). uPAR and MRJ proteins are co-expressed in all four cell lines. The breast cancer cell line MDA-MB-231 showed the highest protein levels for both uPAR and MRJ proteins and was therefore selected for further studies.
Co-immunoprecipitation of UPAR and MRJ in HEK293/uPAR cells transfected with MRJ, and in the breast cancer cell line $M D A-M B-231$. To determine whether interactions between uPAR and MRJ also occur within a cellular context, coIP analyses were carried out using HEK293/uPAR cells that express high levels of uPAR. In this instance, the cells were transfected with myc-tagged MRJ-F for $48 \mathrm{~h}$ before the cells were lysed and subjected to IP with anti-uPAR or anti-myc antibody. As shown in Fig. 5A, three major bands were again evident after probing with anti-uPAR antibody as described above. These uPAR bands were detected in those samples immunoprecipitated with anti-uPAR or anti-myc antibodies, or whole cell lysate, but were either not or more weakly detected in the respective IgG isotype controls, indicating a specific interaction between UPAR and MRJ. After probing with anti-myc antibody (Fig. 5B), a myc-tagged MRJ-F protein band detected in the whole cell lysate and in samples immunoprecipitated with anti-uPAR but not with its isotype goat IgG control. The two bands detected after IP with anti-myc antibody correspond to endogenous c-myc (65 kDa) and myc-tagged MRJ-F protein (32 kDa), but these were not immunoprecipitated by the isotype rabbit $\mathrm{IgG}$ antibody. The coIP of MRJ-F and UPAR in the HEK293/uPAR cell line provides evidence for an interaction between these two proteins in cultured cells. 
A

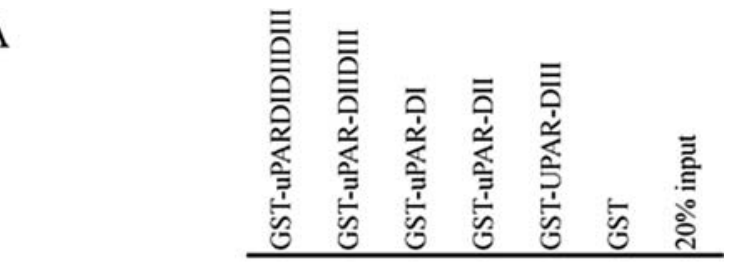

${ }^{35} \mathrm{~S}-\mathrm{MRJ}-\mathrm{F} \rightarrow-\ldots$

B

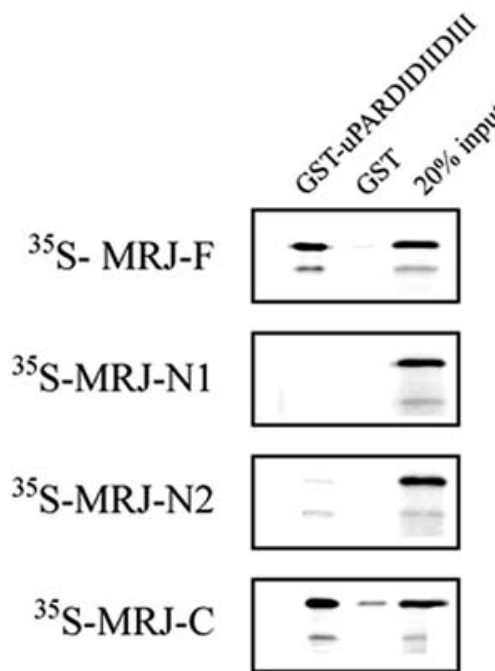

Figure 3. In vitro confirmation of binding of uPAR to MRJ by GST-pull down assays. (A) In vitro $\left[{ }^{35} \mathrm{~S}\right]$-methionine labelled MRJ-F protein was incubated with five GST-uPAR domain constructs. Although all separate UPAR constructs bound MRJ-F, there was increased binding by uPARDIDIIDIII and uPAR-DI baits. (B) Determination of the MRJ region required for binding uPAR. Four $\left[{ }^{35} \mathrm{~S}\right]-$ methionine labelled MRJ proteins (MRJ-F, MRJ-N1, MRJ-N2 and MRJ-C) were incubated with GST-tagged uPARDIDIIDIII. Bound proteins were separated by SDS-PAGE and visualized by autoradiography. The experiment was repeated three times and representative data are shown (20\% input corresponds to $20 \%$ of the total $\left[{ }^{35} \mathrm{~S}\right]$-methionine protein used in the pull down assays).

As shown in Fig. 6, to detect the interaction between endogenously expressed UPAR and MRJ, coIP was performed using breast cancer MDA-MB-231 cells that express both uPAR and MRJ proteins. The UPAR protein was detected in lysate immunoprecipitated with anti-MRJ antibody, but not after coIP with the isotype mouse IgG control antibody (Fig. 6A). The MRJ protein was also detected after IP with anti-uPAR, but not after coIP with goat IgG control antibody (Fig. 6B). To determine whether uPAR specifically bound to MRJ (DNAJB6), MDA-MB-231 lysate was immunoprecipitated with DNAJB4, an antibody for another MRJ family member. After probing with anti-uPAR antibody (Fig. 6A), no uPAR proteins were detected. Similarly, MDAMB-231 lysate was immunoprecipitated with anti-uPAR and probed with anti-DNAJB4 antibody (Fig. 6C), no DNAJB4 proteins were detected suggesting that the DNAJB4 protein may not bind to uPAR under these conditions. This experiment was an additional control to the standard IgG controls to determine specificity of the interaction between UPAR and MRJ. The coIP of uPAR and MRJ in MDA-MB-231 cells indicates that these proteins interact with each other when they express endogenously.
A<smiles>[AlH2]</smiles>

(2.5 kb)

(1.6 kb)

uPAR (1.4 kb)

$18 \mathrm{~S}$ rRNA (2.0 kb)

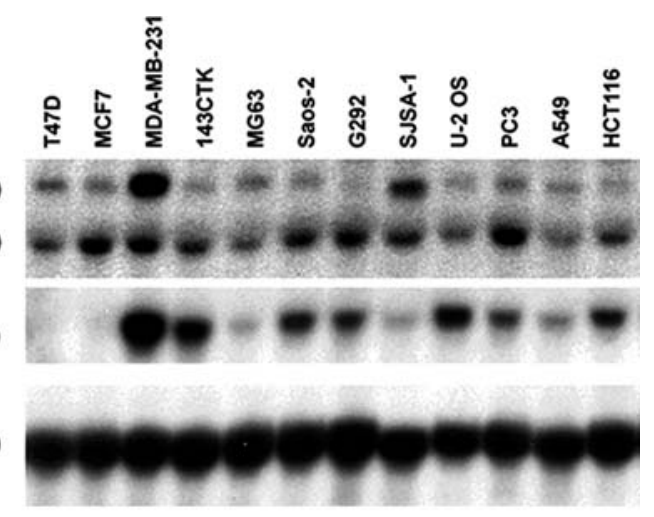

$\mathrm{B}$

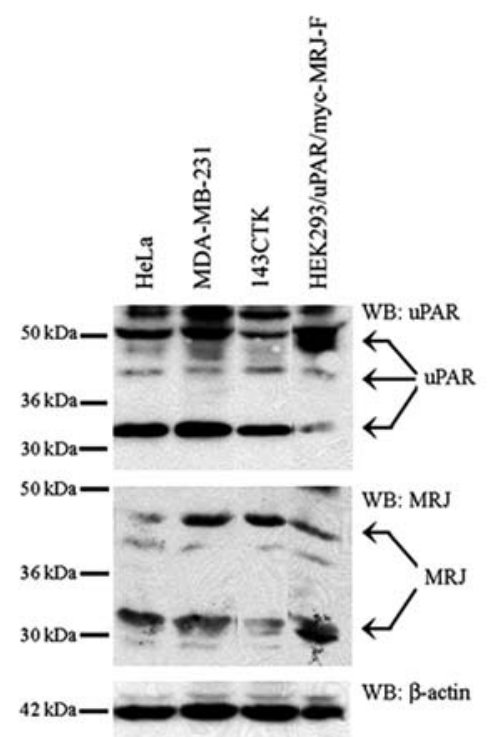

Figure 4. Expression of uPAR and MRJ in cell lines. (A) Northern blot analyses using cancer cell lines. Total RNA (20 $\mu \mathrm{g} /$ lane) was isolated and separated on an agarose-formaldehyde gel and subjected to Northern blotting. MRJ has two transcripts of 2.5 and $1.6 \mathrm{~kb}$, and uPAR a single transcript at $1.4 \mathrm{~kb}$. The $18 \mathrm{~S}$ rRNA was used as a control for equal loading. (B) Western blot analyses of whole cell lysates from a panel of four cell lines. The HEK293/uPAR/myc-MRJ-F cell lysate was used as a positive control to visualise both uPAR and MRJ protein bands. Western blotting for uPAR showed two predominant bands at $\sim 55$ and $35 \mathrm{kDa}$ and a third minor band at $\sim 45 \mathrm{kDa}$ corresponding to glycosylated, deglycosylated and soluble uPAR respectively. The Western blotting for MRJ showed two predominant bands at $\sim 32$ and $\sim 45 \mathrm{kDa}$ which correspond to the two MRJ transcripts of 1.6 and $2.5 \mathrm{~kb}$ seen in the Northern blotting, respectively. $\beta$-actin $(42 \mathrm{kDa})$ was used as a loading control.

MRJ-F increases UPAR-dependent cellular adhesion to vitronectin. To test the biological significance of the UPAR-MRJ interaction, we next examined whether MRJ regulated uPARdependent adhesion to the ligand vitronectin in cell lines HEK293/uPAR and uPAR-deficient HEK293. Previous reports showed that UPAR is involved in adhesion of endothelial and U937 cells to vitronectin $(24,25)$. We therefore evaluated the capacity of MRJ to regulate adhesion of the HEK293/uPAR cells to vitronectin. Initial experiments were carried out to determine the adhesion of HEK293/uPAR and HEK293 cells to either vitronectin or fibronectin, where uPAR-expressing HEK293 cells adhere avidly to Vn (via Vn-binding site of uPAR) and adhere poorly to fibronectin (Fn), whereas uPAR-deficient HEK293 cells adhere to Fn (via the classic 
A

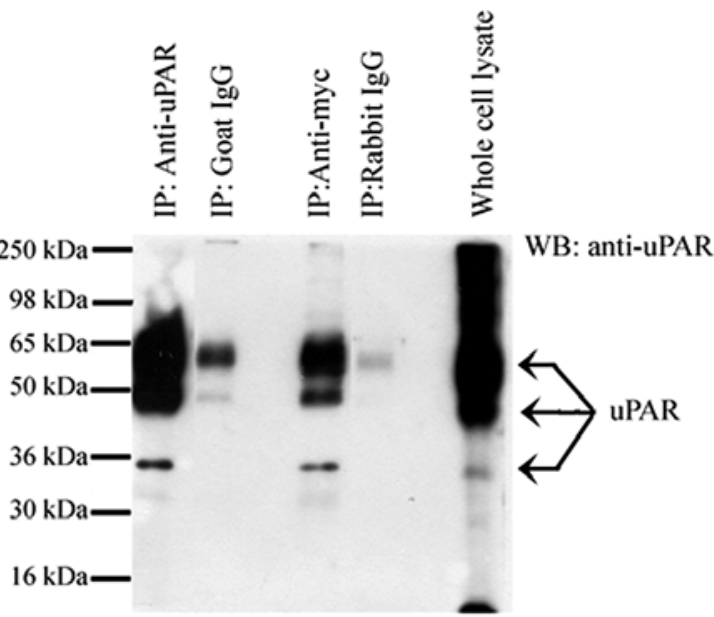

B

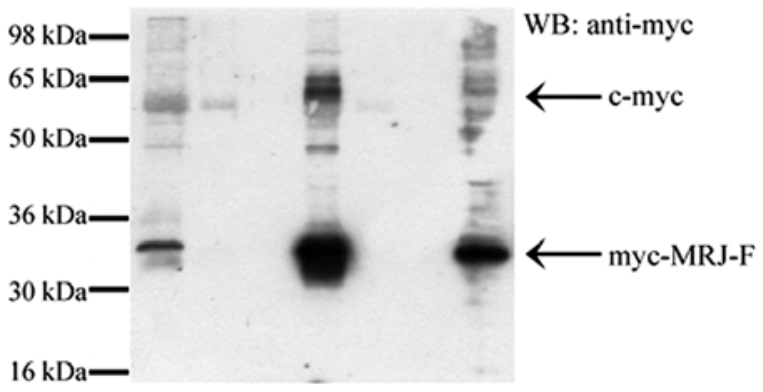

Figure 5. Co-immunoprecipitation of UPAR and MRJ in HEK293/uPAR cells transfected with the myc-tagged MRJ-F construct. (A) Western blotting probed with anti-uPAR antibody. The uPAR protein bands $(35,45$ and $55-60 \mathrm{kDa}$ ) are evident after IP with anti-uPAR or anti-myc, but not with their antibody isotype controls. (B) Western blotting probed with anti-myc antibody. The myc-tagged MRJ-F protein was detected after IP with anti-uPAR or anti-myc, but not their antibody isotype controls. Whole cell lysates $(40 \mu \mathrm{g})$ of transfected HEK293/uPAR cells are shown as a control.

integrin receptor $\alpha 5 \beta 1$ ) and not to Vn. As shown in Fig. 7A, the adhesion potential to vitronectin of HEK293/uPAR cells transfected with MRJ-F was significantly increased compared with control vector PRK5 $(\mathrm{p}<0.01)$. However, no adhesion change was evident for the uPAR negative wild-type HEK293 cells transfected with MRJ-F. On the contrary, when tested on the fibronectin coated plates (Fig. 7B), the adhesion of HEK293/uPAR cells transfected with MRJ-F was less than the cells treated with PRK5 control vector $(\mathrm{p}<0.01)$, and there was no significant different in adhesion between the different treated wild-type HEK293 cells.

To determine whether the increase in adhesion to vitronectin was dependent on the $\mathrm{C}$-terminal region critical of the interaction with uPAR, HEK293/uPAR cells were transfected with full-length MRJ (MRJ-F), or its mutant containing only C-terminal domain (MRJ-C), or its mutant containing only $\mathrm{N}$-terminal domain (MRJ-N1), or vector control (PRK5) (Fig. 7C). Moreover, to determine that the observed increase in adhesion to $\mathrm{Vn}$ is indeed due to uPAR, cells were also incubated with the specific blocking anti-domain I-uPAR antibody (R3). As shown in Fig. 7C, after transfection with MRJ-F, there was an $\sim 50 \%$ increase in the number of adherent cells compared to the PRK5 transfected control $(\mathrm{p}<0.01)$. This increase in adhesion is predominantly due to uPAR as
A

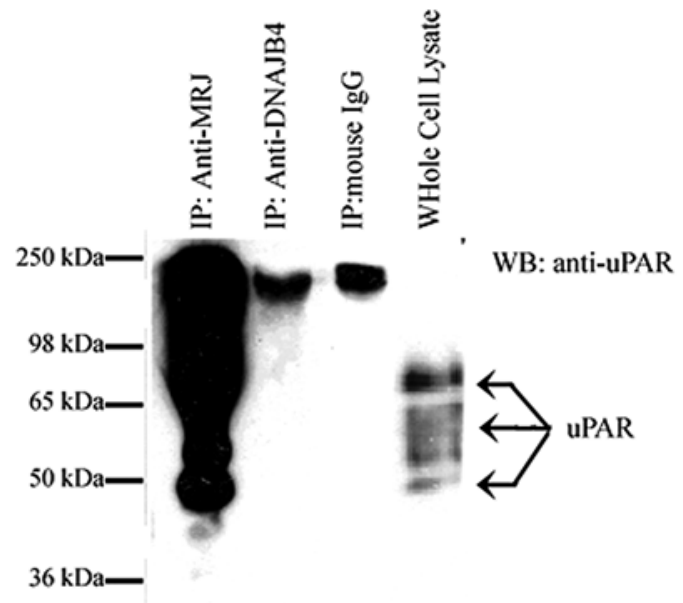

B

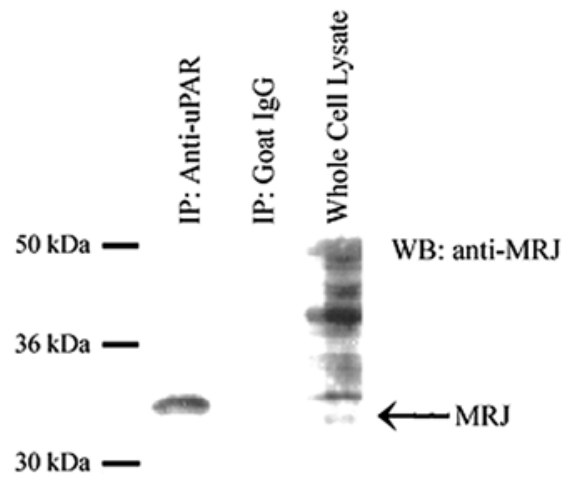

$\mathrm{C}$

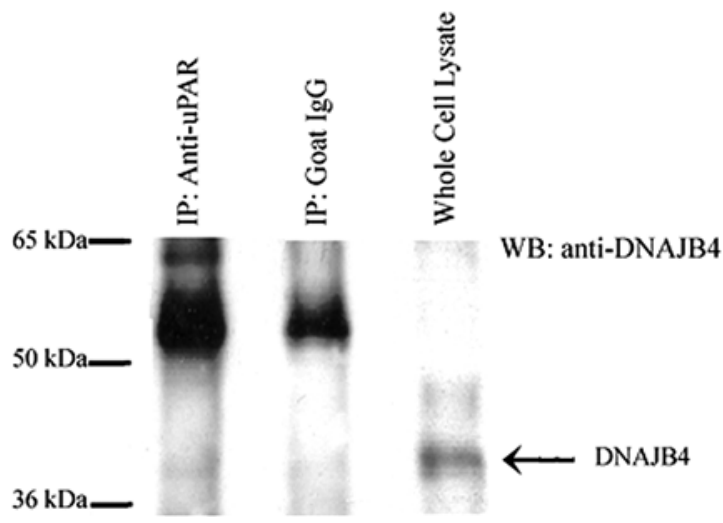

Figure 6. Co-immunoprecipitation of endogenously expressed uPAR and MRJ in breast cancer cells MDA-MB-231. (A) Western blotting probed with anti-uPAR antibody. uPAR protein was detected after IP with anti-MRJ antibody but not with anti-DNAJB4 or mouse IgG isotype control antibodies. The whole cell lysates $(40 \mu \mathrm{g})$ of MDA-MB-231 cells are shown as a control. (B) Western blotting probed with anti-MRJ antibody. MRJ protein was detected after IP with anti-uPAR antibody, but not isotype control goat IgG antibody. The whole cell lysates ( $40 \mu \mathrm{g}$ ) of MDA-MB-231 cells are shown as a control. (C) Western blotting probed with anti-DNAJB4 antibody. DNAJB4 protein was not detected after IP with anti-uPAR antibody. The whole cell lysates $(40 \mu \mathrm{g})$ of MDA-MB-231 cells are shown as a control.

it can be reduced to basal levels by R3, an anti-domain I uPAR specific antibody. There were no significant changes in adhesion compared to the vector control when cells were transfected with either MRJ-C or MRJ-N1 construct suggesting that MRJ-C and MRJ-N1 proteins that contain $\mathrm{C}$-terminal domain only or lack the $\mathrm{C}$-terminal domain respectively, did not have the ability to increase adhesion to vitronectin. 

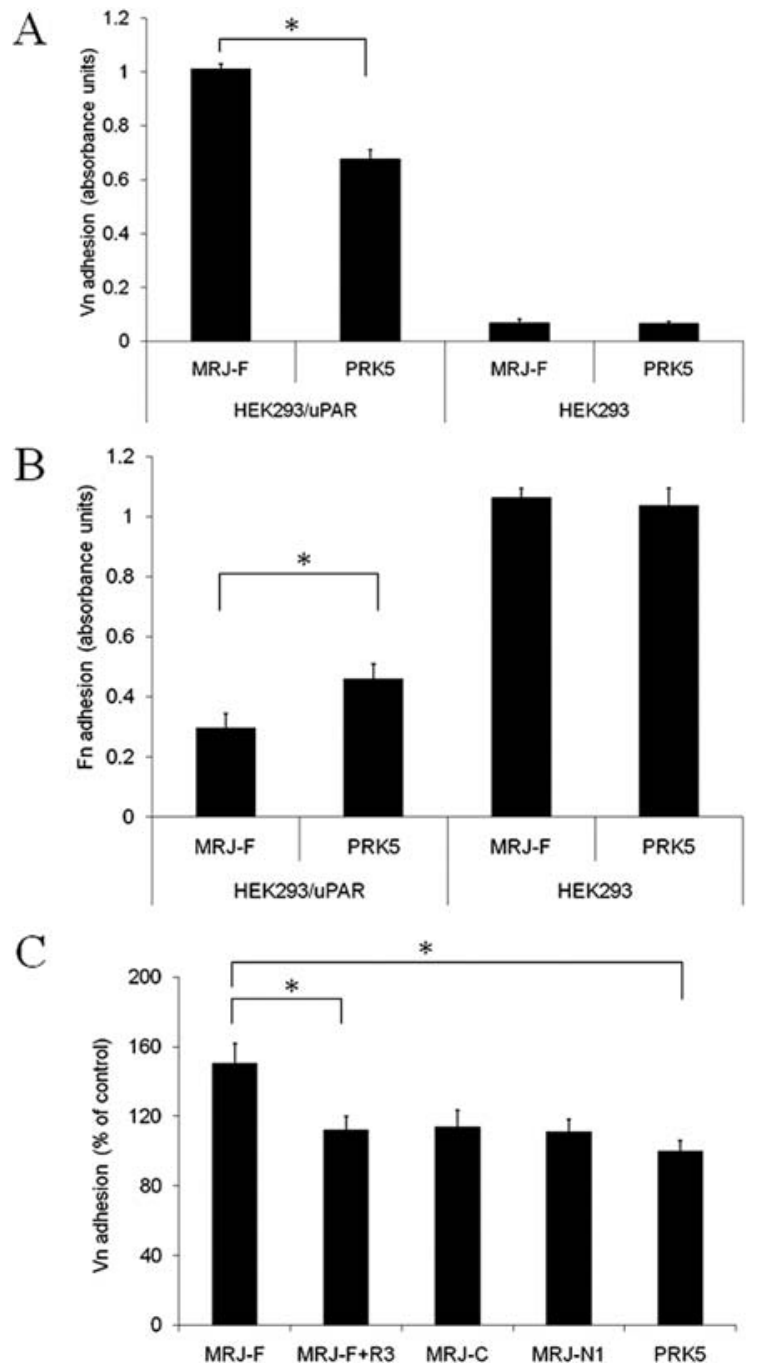

Figure 7. Adhesion assays in HEK293/uPAR and wild-type HEK293 cells (A) Adhesion of HEK293 and HEK293/uPAR cells transfected with full-length MRJ (MRJ-F) or vector control (PRK5) to vitronectin $(2 \mu \mathrm{g} / \mathrm{ml})$. (B) Adhesion of HEK293 and HEK293/uPAR cells transfected with full-length MRJ (MRJ-F) or vector control (PRK5) to fibronectin $(5 \mu \mathrm{g} / \mathrm{ml})$. (C) HEK293/ uPAR cells were transfected with MRJ-F, or its mutant containing only Cterminal domain (MRJ-C), or its mutant containing only N-terminal domain (MRJ-N1), or vector control (PRK5) as shown in the figure. Full length MRJ was also incubated in the presence of the anti-domain I uPAR specific antibody R3 (MRJ-F + R3). Bars indicate the values expressed as the mean \pm standard deviation. Statistical evaluation showed that the effect of MRJ-F in increasing cell adhesion was significant as shown $\left({ }^{*} \mathrm{p}<0.01\right)$.

\section{Discussion}

In this study, five truncated bait constructs of uPAR were designed and used in the yeast two-hybrid system. The screening of a breast cancer cDNA library identified a number of potential candidate proteins that interacted with UPAR, in particular the heat shock protein (HSP) MRJ, a co-chaperon of HSP70. Here, we report for the first time that UPAR and MRJ interact with one another both in vitro and in vivo, resulting in enhanced uPAR-dependent adhesion to vitronectin.

The MRJ protein is member 6 of the DnaJ/HSP40 homolog subfamily B (DnaJB6) (NM_005494). There are two isoforms of MRJ, with the shorter B-isoform (UniProtKB/Swiss-Prot entry O75190-2) homologous to the sequence identified in the yeast two-hybrid screen. The DnaJ family of proteins work together with the DnaK/HSP70 class of chaperones to assist in a wide array of protein folding processes in almost all cellular compartments (26). It has been found to be essential for embryonic development, as homozygous MRJ mutant mice die at mid-gestation due to the failure of chorioallantoic fusion (27). The specific nature of the MRJ mutant phenotype, despite several DnaJ-related genes being expressed in the placenta, suggested that these proteins do not have redundant functions (27). MRJ has also been found to be highly expressed in the brain and over-expression of MRJ in an in vitro Huntington disease model suppressed polyglutamine-dependent protein aggregation, caspase activity and cellular toxicity (28). More recently, the larger isoform of MRJ has been shown to negatively regulate tumour growth in breast cancer with the shorter version also speculated to modulate tumour growth (29).

In our yeast two-hybrid screening, MRJ was identified as a potential new interacting protein of UPAR for both the fulllength uPAR-DIDIIDIII and UPAR-DI baits. The interaction was confirmed using a cross-mating approach (Fig. 2). From the GST-pull down studies, the C-terminal end of MRJ was confirmed to bind specifically to UPAR, independent of either the N-terminal J-domain or the glycine/phenylalanine-rich domain. Further characterization found that MRJ bound with the greatest affinity to GST-tagged full-length uPAR-DIDIIDIII followed by the GST-tagged uPAR-DI (Fig. 3). These data suggest that the interaction between the two proteins occurs mostly via UPAR domain I and the MRJ C-terminal domain. In the MDA-MB-231 and HEK293/uPAR cell lines either endogenous or transfected UPAR/MRJ proteins were able to co-IP (Figs. 5 and 6) confirming that the two proteins interacted in the cells.

HSPs protect cells from harmful conditions, including temperature elevation (heat shock), decrease in $\mathrm{pH}$, oxidative stress and inflammation. Under non-stress conditions, they have multiple housekeeping functions including folding and translocating newly synthesized proteins, as well as activating signaling molecules. Recent findings indicate that cancer cells express high levels of HSPs which are closely correlated with poor prognosis (30). MRJ is an essential co-chaperone of HSP70, with the N-terminal J-domain necessary for its interaction with HSP70 and its chaperone activity $(31,32)$. In breast cancer, similar to uPAR, HSP70 expression is correlated with metastasis and poor prognosis (33). We show here that the important $\mathrm{UPAR} / \mathrm{vitronectin}$ interaction is also at least in part regulated by MRJ (Fig. 7). uPA- and its receptor-dependent cell adhesion to the extracellular matrix protein vitronectin is an important event in wound healing, tissue remodeling, immune response, and cancer. Previous reports showed that on the surface of endothelial and U937 cells, uPAR can mediate cell adhesion to vitronectin $(24,25)$. Vitronectin also provides a substratum for the adhesion of cells in response to inflammation and injury (34). The interaction between vitronectin and UPAR has also been implicated in regulating processes necessary for endothelial cell invasion and migration at vitronectin-rich extracellular matrix sites as well as facilitating intracellular signaling (25). There is also now evidence that vitronectin in liver tumours can support the recruitment and retention of effector lymphocytes by a uPAR-dependent 
mechanism (35). Functionally the interaction between uPAR and vitronectin can promote both cellular adhesion and migration $(24,25,36)$ and may direct UPAR to focal contacts $(37,38)$. The exact mechanism of how MRJ regulates UPAR to increase adhesion to vitronectin is currently being investigated. It has already been shown that the expression of uPAR in HEK293 cells leads to the formation of tight complexes with $\beta 1$ integrins to promote the adhesion of these cells to vitronectin $(39,40)$. Human MRJ was also found to directly interact with the intermediate filament keratin 18 (K18), and regulated K8/18 filament organization (20). Indeed, both uPAR and PAI-1 have also been found to interact with keratin $18(17,41)$ and, MRJ has the ability to direct keratin 18 filaments to the proteasome (42). Furthermore, with the recent report highlighting the role of the longer MRJ isoform in breast cancer (29), these current studies offer a novel insight in offering a potential for the MRJ/uPAR interaction to modulate tumor growth by either altering keratin filament organization and/or promotion in vitronectin binding via $\beta 1$ integrins. Further functional analyses of the novel interaction between UPAR and MRJ and its role in cell adhesion will provide more understanding of their respective roles in regulating cancer metastasis.

\section{Acknowledgements}

We thank Ai-Wei Quan for her technical assistance, Professor David Morris for his financial assistance and co-supervision to Z.L. and A.M. Professor Zichun Hua for support and helpful discussion. Professor G.A.C. Murrell for providing laboratory space, equipment, co-supervision, funding of the experiments and initial $\mathrm{PhD}$ scholarship for C.E.D.B., which experimental work forms a substantial portion of the work published in this report. We are grateful for Jose Perdomo for critical review of the manuscript. This work was supported by the St. George Hospital/South Eastern Sydney \& Illawarra Area Health Service, and the PhD scholarship to C.E.D.B. from the Foundation for Research Science and Technology, New Zealand as well as grants to Y.W. from the University of New South Wales, the Australian Kidney Foundation and the Arthritis Foundation of Australia.

\section{References}

1. Andreasen PA, Egelund R and Petersen HH: The plasminogen activation system in tumor growth, invasion, and metastasis. Cell Mol Life Sci 57: 25-40, 2000

2. Wang Y, Dang J, Johnson LK, Selhamer JJ and Doe WF: Structure of the human urokinase receptor gene and its similarity to CD59 and the Ly-6 family. Eur J Biochem 227: 116-122, 1995

3. Blasi F: Proteolysis, cell adhesion, chemotaxis, and invasiveness are regulated by the u-PA-u-PAR-PAI-1 system. Thromb Haemost 82: 298-304, 1999

4. Blasi F and Carmeliet P: uPAR: a versatile signalling orchestrator. Nat Rev Mol Cell Biol 3: 932-943, 2002.

5. Ossowski L and Aguirre-Ghiso JA: Urokinase receptor and integrin partnership: coordination of signaling for cell adhesion, migration and growth. Curr Opin Cell Biol 12: 613-620, 2000.

6. Nguyen DH, Webb DJ, Catling AD, et al: Urokinase-type plasminogen activator stimulates the Ras/extracellular signalregulated kinase (ERK) signaling pathway and $\mathrm{MCF}-7$ cell migration by a mechanism that requires focal adhesion kinase, Src, and Shc. Rapid dissociation of GRB2/Sps-Shc complex is associated with the transient phosphorylation of ERK in urokinase-treated cells. J Biol Chem 275: 19382-19388, 2000.
7. Behrendt N, Jensen ON, Engelholm LH, Mortz E, Mann M and Dano K: A urokinase receptor-associated protein with specific collagen binding properties. J Biol Chem 275: 1993-2002, 2000.

8. Kiyan J, Kiyan R, Haller H and Dumler I: Urokinase-induced signaling in human vascular smooth muscle cells is mediated by PDGFR-beta. EMBO J 24: 1787-1797, 2005

9. Selleri C, Montuori N, Ricci P, et al: Involvement of the urokinase-type plasminogen activator receptor in hematopoietic stem cell mobilization. Blood 105: 2198-2205, 2005.

10. Wei Y, Tang CH, Kim Y, et al: Urokinase receptors are required for alpha 5 beta 1 integrin-mediated signaling in tumor cells. J Biol Chem 282: 3929-3939, 2007.

11. Llinas P, Le Du MH, Gardsvoll H, et al: Crystal structure of the human urokinase plasminogen activator receptor bound to an antagonist peptide. EMBO J 24: 1655-1663, 2005.

12. Mongiat M, Otto J, Oldershaw R, Ferrer F, Sato JD and Iozzo RV: Fibroblast growth factor-binding protein is a novel partner for perlecan protein core. J Biol Chem 276: 10263-10271, 2001.

13. Aho $\mathrm{S}$ and Uitto $\mathrm{J}$ : Two-hybrid analysis reveals multiple direct interactions for thrombospondin 1. Matrix Biol 17: 401-412, 1998.

14. Mongiat M, Mungiguerra G, Bot S, et al: Self-assembly and supramolecular organization of EMILIN. J Biol Chem 275: 25471-25480, 2000.

15. Bein K and Simons M: Thrombospondin type 1 repeats interact with matrix metalloproteinase 2. Regulation of metalloproteinase activity. J Biol Chem 275: 32167-32173, 2000.

16. Boncela J, Papiewska I, Fijalkowska I, Walkowiak B and Cierniewski CS: Acute phase protein alpha 1-acid glycoprotein interacts with plasminogen activator inhibitor type 1 and stabilizes its inhibitory activity. J Biol Chem 276: 35305-35311, 2001.

17. Magdolen U, Schroeck F, Creutzburg S, Schmitt M and Magdolen V: Non-muscle alpha-actinin-4 interacts with plasminogen activator inhibitor type-1 (PAI-1). Biol Chem 385: 801-808, 2004.

18. Byrne JA, Nourse CR, Basset P and Gunning P: Identification of homo- and heteromeric interactions between members of the breast carcinoma-associated D52 protein family using the yeast two-hybrid system. Oncogene 16: 873-881, 1998.

19. Semple JI, Prime G, Wallis LJ, Sanderson CM and Markie D: Two-hybrid reporter vectors for gap repair cloning. Biotechniques 38: 927-934, 2005 .

20. Izawa I, Nishizawa M, Ohtakara K, Ohtsuka K, Inada H and Inagaki M: Identification of Mrj, a DnaJ/Hsp40 family protein, as a keratin $8 / 18$ filament regulatory protein. J Biol Chem 275: 34521-34527, 2000.

21. Walhout AJ and Vidal M: High-throughput yeast two-hybrid assays for large-scale protein interaction mapping. Methods 24: 297-306, 2001

22. De Bock CE, Lin Z, Itoh T, Morris D, Murrell G and Wang Y: Inhibition of urokinase receptor gene expression and cell invasion by anti-uPAR DNAzymes in osteosarcoma cells. FEBS J 272: 3572-3582, 2005

23. Wei Y, Eble JA, Wang Z, Kreidberg JA and Chapman HA: Urokinase receptors promote betal integrin function through interactions with integrin alpha3beta1. Mol Biol Cell 12: 2975-2986, 2001

24. Waltz DA, Natkin LR, Fujita RM, Wei Y and Chapman HA: Plasmin and plasminogen activator inhibitor type 1 promote cellular motility by regulating the interaction between the urokinase receptor and vitronectin. J Clin Invest 100: 58-67, 1997.

25. Kanse SM, Kost C, Wilhelm OG, Andreasen PA and Preissner KT: The urokinase receptor is a major vitronectin-binding protein on endothelial cells. Exp Cell Res 224: 344-353, 1996.

26. Bukau B and Horwich AL: The Hsp70 and Hsp60 chaperone machines. Cell 92: 351-366, 1998.

27. Hunter PJ, Swanson BJ, Haendel MA, Lyons GE and Cross JC: Mrj encodes a DnaJ-related co-chaperone that is essential for murine placental development. Development 126: 1247-1258, 1999.

28. Chuang JZ, Zhou H, Zhu M, Li SH, Li XJ and Sung CH: Characterization of a brain-enriched chaperone, MRJ, that inhibits Huntingtin aggregation and toxicity independently. J Biol Chem 277: 19831-19838, 2002.

29. Mitra A, Fillmore RA, Metge BJ, et al: Large isoform of MRJ (DNAJB6) reduces malignant activity of breast cancer. Breast Cancer Res 10: R22, 2008. 
30. Calderwood SK, Khaleque MA, Sawyer DB and Ciocca DR: Heat shock proteins in cancer: chaperones of tumorigenesis. Trends Biochem Sci 31: 164-172, 2006.

31. Dai YS, Xu J and Molkentin JD: The DnaJ-related factor $\mathrm{Mr}$ interacts with nuclear factor of activated T cells $\mathrm{c} 3$ and mediates transcriptional repression through class II histone deacetylase recruitment. Mol Cell Biol 25: 9936-9948, 2005.

32. Kelley WL: The J-domain family and the recruitment of chaperone power. Trends Biochem Sci 23: 222-227, 1998

33. Zylicz M, King FW and Wawrzynow A: Hsp70 interactions with the p53 tumour suppressor protein. EMBO J 20: 4634-4638, 2001.

34. Gailit J and Clark RA: Wound repair in the context of extracellular matrix. Curr Opin Cell Biol 6: 717-725, 1994.

35. Edwards S, Lalor PF, Tuncer C and Adams DH: Vitronectin in human hepatic tumours contributes to the recruitment of lymphocytes in an alpha $\mathrm{v}$ beta3-independent manner. Br J Cancer 95: 1545-1554, 2006.

36. Stahl A and Mueller BM: Melanoma cell migration on vitronectin: regulation by components of the plasminogen activation system. Int J Cancer 71: 116-122, 1997.
37. Sitrin RG, Todd RF III, Albrecht E and Gyetko MR: The urokinase receptor (CD87) facilitates CD11b/CD18-mediated adhesion of human monocytes. J Clin Invest 97: 1942-1951, 1996.

38. Madsen CD and Sidenius N: The interaction between urokinase receptor and vitronectin in cell adhesion and signalling. Eur J Cell Biol 87: 617-629, 2008.

39. Wei Y, Lukashev M, Simon DI, et al: Regulation of integrin function by the urokinase receptor. Science 273: 1551-1555, 1996.

40. Tang $\mathrm{CH}$ and Wei Y: The urokinase receptor and integrins in cancer progression. Cell Mol Life Sci 65: 1916-1932, 2008.

41. Busso N, Masur SK, Lazega D, Waxman S and Ossowski L: Induction of cell migration by pro-urokinase binding to its receptor: possible mechanism for signal transduction in human epithelial cells. J Cell Biol 126: 259-270, 1994.

42. Watson ED, Geary-Joo C, Hughes M and Cross JC: The Mrj cochaperone mediates keratin turnover and prevents the formation of toxic inclusion bodies in trophoblast cells of the placenta. Development 134: 1809-1817, 2007. 